\title{
CONSTRUCCIÓN PRELIMINAR DE UN CUESTIONARIO SOBRE CONOCIMIENTOS EN VIH/SIDA EN EXCOMBATIENTES DE COLOMBIA
}

\author{
Ángela Segura-Cardona ${ }^{1, a}$, Dedsy Berbesí-Fernández ${ }^{1, b}$, Doris Cardona-Arango ${ }^{1, c}$, \\ Jaime Ordóñez-Molina ${ }^{1, d}$
}

\begin{abstract}
RESUMEN
Para determinar el nivel de conocimientos sobre VIH/SIDA que tenían excombatientes colombianos en el año 2009, se delineó, construyó y validó un cuestionario, utilizando un diseño mixto, para ese fin se desarrollaron las siguientes etapas:1) Revisión bibliográfica y construcción de los reactivos del cuestionario, discutidos en un grupo focal, 2) Evaluación de la validez de contenido a través de panel de expertos, 3) Aplicación del cuestionario final, donde se seleccionó por conveniencia a 323 personas que pertenecían a un grupo de excombatientes de Colombia, y 4) Validación del cuestionario a través de la evaluación de la consistencia interna y análisis de componentes principales. Como resultados, encontramos que el cuestionario indagaba tres factores: formas de infección, creencias erróneas, y prevención del VIH, que explicaron el $52 \%$ de la varianza. La encuesta tuvo una consistencia interna aceptable (alfa de Cronbach $=0,77$ ). Estos resultados sugieren el uso del cuestionario para evaluar conocimientos relacionados con la forma de infección, conocimientos erróneos y prevención del VIH/SIDA en esta población.
\end{abstract}

Palabras clave: Estudios de validación; Infecciones por VIH; VIH; Grupos vulnerables; Sexo inseguro; Personal militar; Cuestionario (fuente: DeCS BIREME).

\section{PRELIMINARY CONSTRUCTION OF A QUESTIONNAIRE ABOUT KNOWLEDGE OF HIV/AIDS IN COLOMBIAN VETERANS}

\begin{abstract}
In order to identify the level of knowledge about HIVIAIDS in Colombia veterans of war in the year 2009, a questionnaire was designed, built and validated, using a mixed design, for which three stages were followed: 1) Bibliographic review and construction of items of the questionnaire using a focus group, 2) Evaluation of content validity by a pannel of experts, 3) APLICACION of the final questionnaire, we selected non-randomly 323 people who were part of group of veterans in Colombia, and 4) Validation of the questionnaire through the evaluation of internal consistency and principal component analysis. We found that the questionnaire explored three factors: forms of infection, inadequate beliefs, and HIV prevention, which accounted for $52 \%$ of the variance. The survey showed adequate internal consistency values (Cronbach's $\alpha=0.77$ ). These results suggest the use of the questionnaire to assess knowledge level related to the form of infection, inaccurate beliefs and prevention of HIV-AIDS in this population.
\end{abstract}

Key words: Validation studies;HIV Infections; Risk groups; unsafe sex; Military personnel; Questionnaires (source: MesH NLM).

\section{INTRODUCCIÓN}

La Organización Mundial de la Salud estimó el 2008 que, aproximadamente 33,4 millones de personas viven con $\mathrm{VIH}{ }^{(1)}$; por lo que se constituye en un problema de salud pública en continua propagación en poblaciones de alto riesgo. La detección temprana de la infección es una estrategia prioritaria para la prevención, y el tratamiento temprano y oportuno de la enfermedad $(2,3)$. Los patrones de VIH evidencian mayor vulnerabilidad entre personas pobres, mujeres y migrantes ${ }^{(4)}$.
Existen evidencias a nivel mundial que los conflictos armados pueden aumentar las violaciones y debilitar la garantía de los derechos sexuales y reproductivos, en especial los relacionados con VIH/SIDA, en poblaciones vulnerables, propiciando mayor exposición al riesgo de adquirir VIH por parte de aquellos que son o han sido miembros de grupos armados o son parte de las comunidades receptoras de los excombatientes ${ }^{(5)}$.

Colombia ha vivido, en los últimos años, un proceso de paz y reconstrucción a través del desarme, desmovilización

\footnotetext{
1 Grupo de Epidemiología y Bioestadística, Universidad CES. Medellín, Colombia.

a Estadística informática, PhD en Epidemiología; ${ }^{b}$ Enfermera, Magíster en Epidemiología; ${ }^{\circ}$ Administradora de Empresas, PhD en Demografía; ${ }^{\text {a }}$ Médico, PhD en Epidemiología
} 
y reintegración de excombatientes. Estos programas implican una transición, tanto para los combatientes que dejan las armas, para los gobiernos que buscan una salida al conflicto, y para las comunidades que reciben a los excombatientes no militares o desmovilizados ${ }^{(6)}$.

Los conocimientos que posea la población excombatiente sobre infecciones de transmisión sexual, son clave para determinar la prevención que puede hacerse, evitando así el contagio de enfermedades como el VIH/SIDA y, para las instituciones de salud, es útil saber qué actitudes y prácticas son propiciadoras de su transmisión para poder hacer una prevención más efectiva.

Se requiere, entonces, contar con instrumentos válidos y confiables, aplicados a poblaciones específicas, que sean de utilidad práctica y metodológica, y que proporcionen aproximaciones para la detección de riesgos y la prevención del VIH en poblaciones expuestas a su transmisión, como los excombatientes.

Si bien existen instrumentos que evalúan conocimientos sobre VIH estos se validaron en población psiquiátrica, hospitalaria o con características no comparables con excombatientes. La inexistencia de un instrumento previo propició el diseño, construcción y validación de un cuestionario para identificar conocimientos en VIH/SIDA, como una herramienta de fácil aplicación en grupos de excombatientes, dentro del desarrollo de la primera fase del proyecto sobre prevalencia de VIH en esta población.

\section{EL ESTUDIO}

El diseño, construcción y validación del cuestionario se realizó utilizando un diseño mixto (cualitativocuantitativo), desarrollado en las siguientes etapas:

1) Construcción de reactivos del cuestionario. Se identificaron los constructos objeto de estudio (el contenido) (7), tomando como referencia estudios que exploraron conocimientos en otras temáticas. Luego se aplicó la técnica de grupo focal con cincoexcombatientes de 18 a 40 años, procedentes de la región del Bajo Cauca, no residentes en la zona, con quienes se buscaba revisar la redacción de los ítems, evaluar su claridad y generar nuevas preguntas a partir de los conocimientos que pudieran identificarse en el grupo. El propósito del grupo fue enriquecer el cuestionario.

Los resultados junto con los datos teóricos encontrados en la revisión bibliográfica y el análisis de estudios previos, conformaron la plataforma sobre la que se construyó el cuestionario de conocimientos en VIH/SIDA, cuyos ítems tenían respuestas dicotómicas (adecuado e inadecuado).

2) Evaluación de validez de contenido. Se seleccionó un panel de expertos con experiencia y reconocida competencia en metodología y conocimiento en el tema; cuatro expertos fueron seleccionados por el manejo disciplinar del tema (dos médicos, un enfermero y un bacteriólogo) y tres expertos metodológicos (estadístico, epidemiólogo y salubrista). Los expertos revisaron de manera independiente el cuestionario, con el fin de mejorar la redacción, la pertinencia, la extensión y para eliminar posibles errores provenientes de la estructura, además de ser parte en la validación del contenido. La revisión cualitativa determinó si las preguntas del cuestionario eran apropiadas, concisas y completas; la fase culminó con la elaboración del cuestionario.

3) Estudio de campo. Se aplicó la versión final del cuestionario a 323 excombatientes que participaron voluntariamente y que eran parte del proceso de reintegración, de un total de 488 personas identificadas por la Alta Consejería para la Reintegración en la región del Bajo Cauca Antioqueño en 2009.

4) Evaluación de consistencia interna y análisis de componentes principales. Se evaluó la confiabilidad a través de la consistencia interna ( $\alpha$ de Cronbach), consideramos los valores mayores de 0,70 como con una consistencia interna aceptable. Luego se realizó un análisis factorial de tipo exploratorio, mediante el método de componentes principales con rotación varimax, previamente se transformaron las variables cualitativas a través del método de cuantificación óptima y se calculó el coeficiente alfa de Cronbach para cada uno de los factores encontrados. Los tres componentes que surgieron se denominaron como formas de infección ( $\alpha=0,749)$, creencias erróneas $(\alpha=0,70)$ y, prevención de VIH y uso de la prueba $(\alpha=0,52)$. Estos análisis se realizaron en el SPSS 10.0.

\section{DESARROLLO DEL CUESTIONARIO}

Construcción de reactivos y cuestionario. Fueron analizados veinte reactivos o afirmaciones para determinar conocimientos, actitudes y prácticas sobre VIH/SIDA, generados en el grupo focal.

Evaluación de la validez de contenido. Se realizaron dos circulaciones del cuestionario con los expertos seleccionados, en la primera revisión no se excluyó ninguna de las preguntas sobre conocimientos en VIH/SIDA y se decidió enviar nuevamente el cuestionario ajustado en 
la redacción y extensión para su aprobación, sin ningún otro ajuste adicional.

Aplicación del cuestionario final. La aplicación del cuestionario se realizó al 66,1\% (323) de la población de excombatientes. Para la aplicación del modelo se incluyeron doce afirmaciones que evaluaban conocimientos, las otras ocho afirmaciones que evaluaban actitudes y prácticas fueron suprimidas.

Análisis factorial exploratorio. Para el análisis de componentes principales, se cumplieron los criterios de adecuación apropiada de la muestra: índice de adecuación de Káiser-Meyer-Olkin=0,781; prueba de esfericidad de Bartlett significativa $X=1141,939$, $p<0,001$; y comunalidad (variabilidad explicada por los factores comunes) entre 0,37 y 0,712 ; por lo que se procedió a realizar este análisis.

\section{ESTUDIO DE CAMPO}

Características demográficas. Se encuestaron a 323 excombatientes con edades entre 19 y 52 años, con edad promedio de 32,5 \pm 7,4 años; el $50 \%$ de ellos con 34 años o menos y un $75 \%$ con 37 años o menos.

El $74,9 \%$ de la población encuestada eran personas de sexo masculino y el $25,1 \%$ de sexo femenino, con una razón de masculinidad de 3:1. El promedio de edad en los hombres fue $33 \pm 7,3$ años y en las mujeres $31 \pm 8,1$ años.

El promedio de años de estudio cursados fue 5,9 $\pm 2,9$ años; la mitad de los encuestados señalaron haber cursado cinco años o menos; el 75 \% manifestó haber cursado hasta ocho años de escolaridad, el mayor nivel educativo encontrado fue de once años de escolaridad, lo que significa que cursaron toda la educación básica secundaria y media vocacional.

\section{CONOCIMIENTOS SOBRE VIH/SIDA}

Formas de infección.Con respecto a las formas de infección identificadas como conocimientos adecuados por los encuestados, aproximadamente ocho de cada diez excombatientes reconocen que el tener relaciones sexuales sin protección, reutilizar o compartir jeringas o cuchillas, el contacto con sangre infectada y el recibir transfusiones, son factores de riesgo.

Creencias erróneas. Aproximadamente cuatro de cada diez consideran que el tener contacto con sudor o saliva de personas infectadas, utilizar un sanitario o ducha que ha sido usado por una persona infectada, así como utilizar cucharas, tenedores, platos o vasos, pueden favorecer la infección del VIH/SIDA. Estos resultados se pueden apreciar en la Tabla 1.

Medidas preventivas.En cuanto a los conocimientos sobre la prevención del VIH/SIDA en la población excombatiente, el $94 \%$ considera que tener conocimientos sobre el VIH/SIDA ayuda a prevenir su infección.La realización de la prueba de detección, es reconocida como parte fundamental de la prevención y control de esta enfermedad según el $85 \%$ de la población, el $90 \%$ tiene conocimiento sobre la existencia de esta prueba. El $85 \%$ considera que recibir tratamiento para la enfermedad ayuda a mejorar sus condiciones de vida y salud.

Tabla 1. Conocimientos sobre VIH/SIDA en excombatientes de Antioquía, Colombia, 2009.

\begin{tabular}{lcc}
\hline \multicolumn{1}{c}{ Conocimientos } & $\begin{array}{c}\text { Adecuados } \\
\%\end{array}$ & $\begin{array}{c}\text { Inadecuados } \\
\%\end{array}$ \\
\hline Formas de infección & 84 & 16 \\
\hline Tener relaciones sexuales con personas infectadas sin utilizar condón & 84 & 16 \\
\hline Utilizar jeringas o cuchillas que ya han sido utilizadas por personas infectadas & 82 & 18 \\
\hline Tener contacto con sangre de otras personas a través de heridas & 83 & 17 \\
\hline Recibir transfusiones de sangre de una persona infectada & 87 & 13 \\
\hline Creencias erróneas & 61 & 39 \\
\hline El contacto con sudor o saliva de personas infectadas & 57 & 43 \\
\hline Utilizar un sanitario o ducha que ha sido usado por una persona infectada & 58 & 42 \\
\hline Utilizar cucharas, tenedores, platos o vasos que han sido usados por personas infectadas & 58 & 42 \\
\hline Los síntomas del VIH/SIDA aparecen a los pocos días de haber sido contagiado & 72 & 28 \\
\hline Prevención de VIH y uso de la prueba & 89 & 11 \\
\hline Realizarse una prueba de VIH puede evitar contagiar a otras personas & 85 & 15 \\
\hline Tener conocimientos sobre el VIH/SIDA ayuda a prevenir su infección & 94 & 6 \\
\hline Existe una prueba que permite saber si una persona tiene VIH/SIDA & 90 & 10 \\
\hline Las personas con VIH/SIDA que reciben un tratamiento pueden mejorar sus condiciones de salud & 85 \\
y vivir durante más tiempo. & & 15 \\
\hline
\end{tabular}




\section{EVALUACIÓN DE COMPONENTES PRINCIPALES Y CONSISTENCIA INTERNA}

Se hallaron tres componentes que explicaron el 52,0 \% de la varianza: formas de infección, creencias erróneas y prevención; los resultados se aprecian en la Tabla 2.

El primero se denominó formas de infección y tuvo un alfa de Cronbach de 0,749; las correlaciones de los ítems que lo conforman con la puntuación total del factor variaron entre 0,518 y 0,690 . Se aglutinan las variables sobre transmisión del VIH: tener relaciones sexuales, reutilizar jeringas, contacto con sangre infectada y recibir transfusiones.

El segundo fue de creencias erróneas, la consistencia interna fue 0,70 y las correlaciones ítem-factor variaron entre 0,558 y 0,685 . Se agruparon variables sobre opiniones erróneas acerca de la transmisión: contacto con sudor, utilización de sanitario y cucharas como formas de transmisión y tener síntomas de $\mathrm{VIH}$ al poco tiempo de ser infectados.

Finalmente, el tercer componente, prevención, se relacionó principalmente con la prueba de $\mathrm{VIH}$, con una consistencia de 0,52 , las correlaciones variaron entre 0,344 y 0,472 . Recogió variables de importancia de la prueba diagnóstica para detección del $\mathrm{VIH}$, tratamiento para aumentar la sobrevida y conocimientos en prevención.

\section{DISCUSIÓN}

Algunos autores han realizado estudios con el fin de determinar conocimientos, actitudes y prácticas en el tema de $\mathrm{VIH} / \mathrm{SIDA}$, especialmente en población adolescente ${ }^{(8-11)}$. En cuanto a población excombatiente no se identificaron estudios tan específicos, a pesar que son una población de riesgo, dada las circunstancias en que ocurren los procesos de reintegración que aumentan la vulnerabilidad a contraer infecciones de transmisión sexual incluyendo el VIH ${ }^{(12)}$.

El presente estudio encontró desinformación en la población de excombatientes, sobre las vías de transmisión del VIH y las formas de protección; similar situación a la observada en el estudio sobre conocimientos, actitudes y prácticas realizado en jóvenes menores de 18 años en Cali-Colombia, donde se encontraron vacíos en los conocimientos sobre las formas de infección y las formas de evitar la infección de VIH/SIDA ${ }^{(13)}$.

En un estudio sobre factores de riesgo de infección por VIH/SIDA y otras infecciones de transmisión sexual, asociados a conocimientos, actitudes y prácticas en universitarios, encontraron que $99 \%$ de los encuestados reconocía las formas de transmisión del VIH/SIDA ${ }^{(14)}$; esta proporción fue menor en la población excombatiente, por lo que se requiere fortalecer los procesos educativos y de prevención, reconociendo las diferencias socioculturales y la heterogeneidad de la población, para el diseño y orientación de nuevas estrategias.

Una vez cumplidos los principales requisitos que se contemplan en la elaboración de un instrumento, tanto en lo que se refiere a su sustento teórico como a su fundamentación psicométrica, de acuerdo con los resultados descritos previamente, la encuesta preliminar

Tabla 2. Componentes principales del conocimiento en VIH/SIDA en excombatientes de Antioquía, Colombia, 2009.

\begin{tabular}{|c|c|c|c|}
\hline Conocimientos & $\begin{array}{l}\text { Formas de } \\
\text { infección }\end{array}$ & $\begin{array}{l}\text { Creencias } \\
\text { erróneas }\end{array}$ & $\begin{array}{l}\text { Prevención } \\
\text { de VIH }\end{array}$ \\
\hline $\begin{array}{l}\text { Tener relaciones sexuales, con personas infectadas, sin utilizar correctamente el } \\
\text { condón. }\end{array}$ & 0,622 & & \\
\hline Utilizar jeringas o cuchillas que ya han sido utilizadas por personas infectadas. & 0,833 & & \\
\hline $\begin{array}{l}\text { Tener contacto con sangre de personas infectadas -cuando nosotros tenemos algún } \\
\text { tipo de herida que entra en contacto con la sangre de esa persona-. }\end{array}$ & 0,729 & & \\
\hline Recibir transfusiones de sangre de una persona infectada. & 0,811 & & \\
\hline El contacto con sudor o saliva de personas infectadas. & & 0,699 & \\
\hline Utilizar un sanitario o ducha que ha sido usado por una persona infectada. & & 0,783 & \\
\hline $\begin{array}{l}\text { Utilizar cucharas, tenedores, platos o vasos que han sido usados por personas in- } \\
\text { fectadas. }\end{array}$ & & 0,780 & \\
\hline Los síntomas de VIH/SIDA aparecen a los pocos días de haber sido contagiados. & & 0,545 & \\
\hline Realizarse una prueba de VIH puede ayudar a prevenir la infección de otras personas. & & & 0,722 \\
\hline Tener conocimientos sobre VIH/SIDA ayuda a prevenir que las personas se contagien. & & & 0,589 \\
\hline Existe una prueba que permite saber si una persona tiene VIH/SIDA. & & & 0,552 \\
\hline $\begin{array}{l}\text { Las personas que reciben tratamiento pueden mejorar sus condiciones de salud y vivir } \\
\text { durante más tiempo. }\end{array}$ & & & 0,563 \\
\hline Alfa de Cronbach & 0,749 & 0,700 & 0,520 \\
\hline
\end{tabular}

Índices factoriales (saturación) de los ítems de la encuesta de conocimientos después de la rotación Varimax con la normalización de Káiser. 
para determinar conocimientos sobre $\mathrm{VIH}$, evidenció: a) claridad y buena discriminación de los reactivos; b) poseer validez de constructo; y c) un alfa de Cronbach que supone que el instrumento tiene buena consistencia interna.

El estudio tuvo algunas limitaciones, como dificultades en el acercamiento a estapoblación, por lo cual la muestra no es representativa y no puede generalizarse los hallazgos; también es necesaria la aplicación del cuestionario en otras poblaciones para validar los constructos emergentes; y en el análisis se podría usar matrices tetracóricas, puesto que el cuestionario tiene preguntas dicotómicas, aspectos que pueden limitar el alcance de estos resultados.

Los resultados muestran que el cuestionario desarrollado en el presente estudio es un instrumento que podría ser aplicado para identificar conocimientos en VIH en población de excombatientes. El análisis factorial confirmó la existencia de tres dimensiones, las cuales se correspondían con las propuestas en el diseño, demostrando de forma preliminar su validez de contenido.

El cuestionario de conocimientos demostró que la consistencia interna al interior de cada componente es aceptable en las dimensiones: formas de infección, medidas preventivas y creencias erróneas en población de excombatientes. Este cuestionario podría ser utilizado tanto en la investigación como en la práctica diaria, una vez se valide en otras poblaciones y se compare con otros instrumentos, pueses un cuestionario corto y de rápida administración, lo que haría más factible su aplicación en la asesoría preprueba de VIH/SIDA y puede ser de gran utilidad para decidir las intervenciones educativas necesarias en la asesoría postprueba.

\section{Contribuciones de autoría}

Todos los autores participaron en la concepción y diseño del trabajo, recolección de datos, análisis estadístico e interpretación de la información y en la redacción del manuscrito. Todos los autores aprobaron la versión final del documento.

\section{Fuentes de financiamiento}

Este estudio fue financiado por la Organización Internacional de Migraciones y por United States Agency International Development (USAID).

\section{Conflictos de interés}

Los autores declaran no tener conflictos de interés.

\section{REFERENCIAS BIBLIOGRÁFICAS}

1. ONUSIDA. Situación de la Epidemia de Sida 2009 [Internet]. Ginebra: ONUSID; [citado el 22 de junio del 2010]. Disponible en: http://www.unaids.org/es/KnowledgeCentre/HIVData/ EpiUpdate/EpiUpdArchive/2009/default.asp

2. Protto JP, Schaaf D, Suárez MF, Darras C. Entorno epidemiológico y respuesta a la epidemia del VIH en Bolivia. Rev Panam Salud Publica. 2008;23(4):288-94.

3. Underhill K, Operario D, Montgomery P. Programas de abstinencia exclusiva para la prevención de la infección por VIH en países de ingresos altos [Internet]. Oxford: The Cochrane Collaboration; [citado el 22 de junio del 2010].Disponible en: http://www2.cochrane.org/reviews/es/ab005421.html

4. Organización Mundial de la Salud. Vigilancia del VIH en segunda generación [Internet]. Ginebra: OMS; [citado el 22 de junio del 2010]. Disponible en: http://whqlibdoc.who.int/ hq/2000/WHO_CDS_CSR_EDC_2000.5_spa.pdf

5. Inter-Agency Standing Committee. Intervenciones relacionadas con el VIH/SIDA en contextos de emergencia [Internet]. Ginebra: ONUSID; [citado el 22 de junio del 2010]. Disponible en: http://data.unaids.org/pub/InformationNote/2003/iascguidelines_es.pdf

6. Theidon K, Betancourt P. Transiciones conflictivas: combatientes desmovilizados en Colombia. Análisis Político. 2006;58(1):92-111.

7. Lamprea JA. Gómez-Restrepo C. Validez en la evaluación de escalas. Rev Colomb Psiquiatr. 2007;36(2):340-48.

8. Macci ML, Benitez L, Corvalan A, Nuñez C, Ortigoza D. Conocimientos, actitudes y practicas acerca del VIH/SIDA en jóvenes de nivel medio de Educación del área metropolitana, Paraguay. Rev Chil Pediatr. 2008;79(2): 206-17.

9. Rassetto M J, Herrera D, Massa M, Ruiz A. Diseño de un cuestionario para identificar actitudes sobre HIVISIDA. VII Congreso Internacional sobre Investigación en la Didáctica de las Ciencias. Facultad de Farmacia de la Universidad de Granada. Granada, España. 7 al 10 de septiembre de 2005.

10. Zometa, CS. Translation, adaptation, and validation of an instrument to evaluate HIVIAIDS knowledge and attitudes for use with Salvadorian high school students (2004) [Tesis Doctoral]. Florida, USA: University of South Florida; 2004.

11. Dávila ME, Tagliaferro AZ, Bullones $X$, Daza D. Nivel de Conocimiento de Adolescentes sobre VIH/SIDA.Rev Salud Pública. 2008;10(5):716-22.

12. Spiegel PB. HIVIAIDS among conflict-affected and displaced populations: dispelling myths and taking action. Disasters. 2004;28:322-39.

13. Mosquera J, Mateus JC.Conocimientos, actitudes y prácticas sobre métodos de planificación familiar, VIH-SIDA y el uso de los medios de comunicación en jóvenes. ColombMed. 2003;34(4):206-12.

14. Vera LM, López N, Ariza N, Díaz LM, Flórez Y, Franco S, et al. Asociación entre el área de estudio y los conocimientos y comportamientos frente a la transmisión del VIH/SIDA en los estudiantes de la Universidad Industrial de Santander. Colomb Med. 2004; 35(2):62-8.

Correspondencia: Ángela María Segura Cardona

Dirección: Universidad CES Calle 10A, 22-04; Medellín, Colombia. Teléfono: 57 (4) 4440555 Ext.1408

Correo electrónico: asegura@ces.edu.co 